\title{
Image Reconstruction by Regularized Nonlinear Inversion-Joint Estimation of Coil Sensitivities and Image Content
}

\author{
Martin Uecker, ${ }^{1 *}$ Thorsten Hohage, ${ }^{2}$ Kai Tobias Block, ${ }^{1}$ and Jens Frahm ${ }^{1}$
}

\begin{abstract}
The use of parallel imaging for scan time reduction in MRI faces problems with image degradation when using GRAPPA or SENSE for high acceleration factors. Although an inherent loss of SNR in parallel MRI is inevitable due to the reduced measurement time, the sensitivity to image artifacts that result from severe undersampling can be ameliorated by alternative reconstruction methods. While the introduction of GRAPPA and SENSE extended MRI reconstructions from a simple unitary transformation (Fourier transform) to the inversion of an illconditioned linear system, the next logical step is the use of a nonlinear inversion. Here, a respective algorithm based on a Newton-type method with appropriate regularization terms is demonstrated to improve the performance of autocalibrating parallel MRI-mainly due to a better estimation of the coil sensitivity profiles. The approach yields images with considerably reduced artifacts for high acceleration factors and/or a low number of reference lines. Magn Reson Med 60:674-682, 2008. (C) 2008 Wiley-Liss, Inc.
\end{abstract}

Key words: inverse problems; iterative reconstruction; parallel imaging; nonlinear inversion

Independent MRI acquisitions from multiple receiver coils may be exploited for encoding part of the spatial information of an object by the spatially varying coil sensitivities. When used in conjunction with conventional phase-encoding by magnetic field gradients, coverage of the k-space for image reconstruction may become undersampled along a suitable phase-encoding dimension which in turn corresponds to a reduction of overall scan time.

To take full advantage of such parallel imaging techniques the information that necessarily needs to be derived from the sensitivities of the different receiver coils has to be known with sufficiently high accuracy. Unfortunately, however, the receiver sensitivities depend on the dielectric properties of the object under investigation and reflect even small object movements. To compensate for these effects, autocalibrating methods have been developed which determine the required information from reference lines in the center of k-space. The reference lines are usually acquired at the same time as the actual object-defining lines in $\mathrm{k}$-space.

The common reconstruction methods for autocalibrated parallel imaging are based on a sequential approach: the

\footnotetext{
${ }^{1}$ Biomedizinische NMR Forschungs $\mathrm{GmbH}$ am Max-Planck-Institut für biophysikalische Chemie, Göttingen, Germany

${ }^{2}$ Institut für Numerische und Angewandte Mathematik, Georg-AugustUniversität, Göttingen, Germany

Presented in part at the 15th Annual Meeting of ISMRM, Berlin, Germany, 2007

*Correspondence to: M. Uecker, Biomedizinische NMR Forschungs $\mathrm{GmbH}$, 37070 Göttingen, Germany. E-mail: muecker@gwdg.de

Received 28 September 2007; revised 21 April 2008; accepted 22 April 2008. DOI 10.1002/mrm.21691

Published online 5 August 2008 in Wiley InterScience (www.interscience.wiley com).

(c) 2008 Wiley-Liss, Inc.
}

determination of the information about the coil sensitivities from the reference lines is followed by the reconstruction of an image by a linear process. As will be explained later such two-step techniques make only suboptimal use of the available data. With the help of an alternating minimization method, Ying and Sheng (1) recently proposed to improve this situation by iteratively optimizing both the coil sensitivities and the image content until a joint solution is found. Extending these ideas, the purpose of this work is to show how a regularized nonlinear inversion technique based on a Newton-type method with appropriate regularization terms provides a generic and convenient framework for solving this problem in the context of MRI reconstruction.

\section{THEORY}

\section{Parallel Imaging}

The MRI signal obtained for multiple receiver coils is given by

$$
s_{j}(t)=\int \mathrm{d} \vec{x} e^{i \vec{k}(t) \vec{x}} \rho(\vec{x}) c_{j}(\vec{x}) \quad j=1, \cdots, N .
$$

Here $\rho$ denotes the proton density and $c_{j}$ the sensitivity profiles of the individual receiver coils. $\vec{k}(t)$ is the chosen k-space trajectory. If the coil sensitivity profiles are known, this equation represents a linear system which can be solved numerically (2). Existing direct methods either utilize the decoupling of the equation in image space for regular sampling patterns like SENSE (3-5) or approximate a sparse inverse by using the k-space locality principle as employed for SMASH $(6,7)$ and its successors. Unfortunately, the MRI signal equation becomes increasingly ill-conditioned for large acceleration (or undersampling) factors. As a consequence, the inversion of the system leads to the amplification of noise that contributes to the left-hand side of the equation. To counter this effect the inversion has to be regularized (2). If the receiver sensitivities are not known, it is common practice to compute the necessary information in a calibration step and then proceed as before (8). For autocalibrating SENSE the coil sensitivity profiles are determined directly from the reference lines (9). For GRAPPA (10) the reconstruction weights are obtained by a fit to the reference lines.

In general, a determination of coil sensitivities from only the center of k-space does not take advantage of all available information. Although the information about a smooth coil profile is mostly localized in the k-space center, the measured data represents the convolution of the coil profiles with the object function which shifts information from the center of k-space to its outer parts. Because even small errors in the sensitivity profiles lead to residual aliasing 
artifacts in the resulting image, an optimal reconstruction should exploit all available k-space data rather than only a small part in its center. This can be accomplished by a nonlinear inversion technique.

\section{Parallel Imaging as Nonlinear Inversion}

To solve for the object function and the sensitivity profiles at the same time, the method proposed here applies a regularized nonlinear inversion to the reconstruction problem. The basic MRI signal equation is understood as a nonlinear operator equation with an operator $F$ which maps the proton density and the coil sensitivity profiles to the measured data

$$
F(x)=y \quad \text { with } \quad x=\left(\begin{array}{c}
\rho \\
C_{1} \\
\vdots \\
C_{N}
\end{array}\right)
$$

This equation is solved by a Newton-type algoritm, more precisely the Iteratively Regularized Gauss Newton Method (IRGNM), see $(11,12)$ as general references. In the following, it is assumed that the operator equation is given in a discretized form where all functions are represented by vectors of point values on a rectangular grid.

\section{Iteratively Regularized Gauss Newton Method}

The general idea of the Newton algorithm is as follows. A linearization of the equation around an initial guess $x_{n}$ yields

$$
F\left(x_{n}+d x\right) \approx D F\left(x_{n}\right) d x+F\left(x_{n}\right) .
$$

Here $D F\left(x_{n}\right)$ denotes the Fréchet derivative (or Jacobian) of $F$ at the point $x_{n}$. This linearized equation is then solved for the update $d x$

$$
D F\left(x_{n}\right) d x+F\left(x_{n}\right)=y .
$$

Under certain conditions updates of $x_{n+1}=x_{n}+d x$ and an iteration of this scheme converges to a solution.

To calculate an approximate solution to the linearized problem the conjugate gradient algorithm can be used. This algorithm needs to be applied to a symmetric matrix, a condition which can be ensured by multiplying both sides with the adjoint of $D F\left(x_{n}\right)$

$$
D F\left(x_{n}\right)^{H} D F\left(x_{n}\right) d x=D F\left(x_{n}\right)^{H}\left(y-F\left(x_{n}\right)\right) .
$$

Because of the bad conditioning of the linearized equations the inversion must be regularized. Adding a positive definite regularization matrix, e.g. the identity, to the matrix $D F\left(x_{n}\right)^{H} D F\left(x_{n}\right)$ yields the well known LevenbergMarquardt algorithm

$$
\left(D F\left(x_{n}\right)^{H} D F\left(x_{n}\right)+\alpha_{n} I\right) d x=D F\left(x_{n}\right)\left(y-F\left(x_{n}\right)\right) .
$$

The regularization parameter $\alpha_{n}$ is reduced in each step. For large values of $\alpha_{n}$ the algorithm is related to the gradient descent algorithm

$$
\left(\alpha_{n} I\right) d x=D F\left(x_{n}\right)^{H}\left(y-F\left(x_{n}\right)\right) .
$$

For low values the algorithm represents the classic GaussNewton method

$$
D F\left(x_{n}\right)^{H} D F\left(x_{n}\right) d x=D F\left(x_{n}\right)^{H}\left(y-F\left(x_{n}\right)\right) .
$$

The Levenberg-Marquardt algorithm takes advantage of both these ideas by using the more robust gradient descent algorithm at the beginning of the iterative process (far from the solution) and the faster Gauss-Newton algorithm at the end (near the solution). Its properties can be understood in a reformulation as a sequence of quadratic optimization problems where the update $d x$ corresponds to the unique minimizer of the functional

$$
\left\|D F\left(x_{n}\right) d x-\left(y-F\left(x_{n}\right)\right)\right\|^{2}+\alpha_{n}\|d x\|^{2} .
$$

Although each individual update is regularized to suppress noise, it is still possible that small residual noise in each update accumulates in the final solution. A more stable algorithm is given by changing the update rule to minimize

$$
\left\|D F\left(x_{n}\right) d x-\left(y-F\left(x_{n}\right)\right)\right\|^{2}+\alpha_{n}\left\|x_{n}+d x-x_{0}\right\|^{2} .
$$

In this case the regularization no longer applies to the update itself but to the result of the update with respect to the initial guess. A suitably revised algorithm, the IRGNM, then relates to an update rule

$$
d x=\left(D F\left(x_{n}\right)^{H} D F\left(x_{n}\right)+\alpha_{n} I\right)^{-1} D F\left(x_{n}\right)^{H}\left(y-F\left(x_{n}\right)+\alpha_{n}\left(x_{n}-x_{0}\right)\right) .
$$

The regularization parameters are always chosen to be of the form $\alpha_{n}=\alpha_{0} q^{n}$ with the same $q \in(0,1)$, usually $q=\frac{2}{3}$.

To apply this IRGNM algorithm to parallel MRI reconstructions, implementations of the operator, its derivative, and the adjoint of the derivative are needed. The operator is given by

$$
F: x \mapsto\left(\begin{array}{c}
P \mathcal{F}\left\{c_{1} \cdot \rho\right\} \\
\vdots \\
P \mathcal{F}\left\{c_{N} \cdot \rho\right\}
\end{array}\right) \quad \text { with } \quad x=\left(\begin{array}{c}
\rho \\
c_{1} \\
\vdots \\
c_{N}
\end{array}\right) \text {, }
$$

where $\mathcal{F}$ is the (multidimensional) Fourier transform and $P$ is the orthogonal projection onto the trajectory which-for cartesian sampling-is a diagonal matrix with ones at the positions of the measured data and zeros elsewhere. The derivative of the operator can easily be calculated by using the linearity of the Fourier transform and the product rule of derivatives

$$
D F(x)\left(\begin{array}{c}
d \rho \\
d c_{1} \\
\vdots \\
d c_{N}
\end{array}\right)=\left(\begin{array}{c}
P \mathcal{F}\left\{\rho \cdot d c_{1}+d \rho \cdot c_{1}\right\} \\
\vdots \\
P \mathcal{F}\left\{\rho \cdot d c_{N}+d \rho \cdot c_{N}\right\}
\end{array}\right) .
$$

Because of the unitarity of $\mathcal{F}$ the adjoint is then given by

$$
D F^{H}(x)\left(\begin{array}{c}
y_{1} \\
\vdots \\
y_{N}
\end{array}\right)=\left(\begin{array}{c}
\sum_{j=1}^{N} c_{j}^{\star} \cdot \mathcal{F}^{-1}\left\{P^{H} y_{j}\right\} \\
\rho^{\star} \cdot \mathcal{F}^{-1}\left\{P^{H} y_{1}\right\} \\
\vdots \\
\rho^{\star} \cdot \mathcal{F}^{-1}\left\{P^{H} y_{N}\right\}
\end{array}\right)
$$

with the star denoting pointwise complex conjugation. 

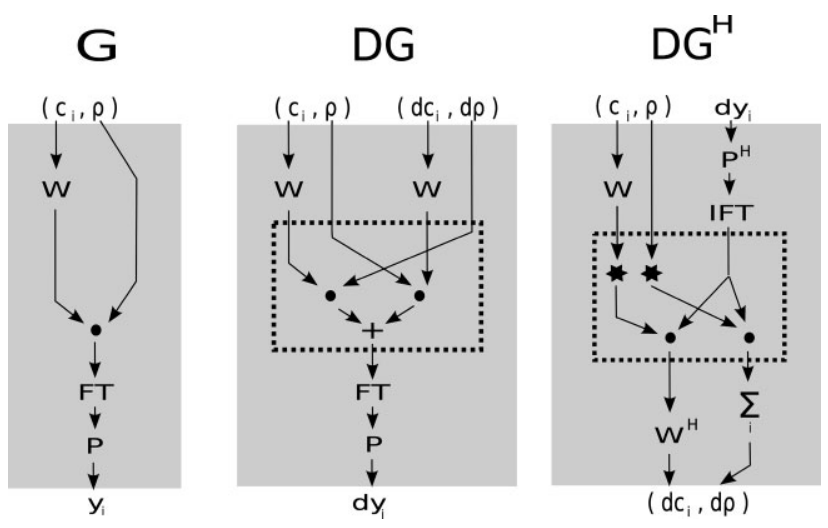

FIG. 1. Flow chart for the calculation of the operator $(G)$, its derivative (DG), and the adjoint of the derivative $\left(D G^{H}\right.$ ) from $c_{i}$ (coil sensitivities) and $\rho$ (proton density). $\mathrm{W}=$ preconditioning matrix, $\mathrm{W}^{\mathrm{H}}$ = adjoint of $\mathrm{W}, \mathrm{FT}=$ Fourier transformation, $\mathrm{IFT}=$ inverse $\mathrm{FT}, \mathrm{P}=$ projection onto the trajectory, $\mathrm{y}_{\mathrm{i}}=$ data, $\cdot=$ pointwise multiplication, + $=$ addition, $\star=$ complex conjugation .

Once the dependency on the coil sensitivities is removed, that is the coil sensitivities are treated as constants, the resulting operator becomes linear while the parts corresponding to the update of the coil sensitivities in the derivative and its adjoint vanish. As a consequence, the algorithm effectively transforms into a conjugate gradient (CG) variant of SENSE, as commonly used for non-cartesian MRI (4). When started with suitable coil sensitivities this modified algorithm produces solutions similar to those obtainable from a CG-SENSE algorithm with decreasing regularization.

\section{Regularization}

A direct application of the described IRGNM algorithm to the problem of autocalibrated parallel imaging would yield an unrealistic solution to the MRI signal equation. Basically, the coil sensitivities would include a substantial portion of the object information and the object part would not be unfolded correctly. The reason is that the equation is highly underdetermined-even in the fully sampled case. This can be seen by the fact that multiplying the object part of a solution $\left(\rho, c_{j}\right)$ by some arbitrary complex function $g$ and dividing the coil sensitivities by the same function gives another solution $\left(\rho \cdot g, c_{j} / g\right)$.

The problem may be overcome by adding a priori knowledge about the object and the coil sensitivities. For example, while the object may contain edges, the coil sensitivities are generally rather smooth. This can be ensured by a smoothness enforcing norm for the coil profiles that is used during the quadratic optimization problems solved in each Newton step. An appropriate choice is a Sobolev norm given by

$$
\|f\|_{l}=\left\|(\mathrm{I}-\Delta)^{I / 2} f\right\|
$$

for some chosen index 1 . The 2D-Laplacian is given by $\Delta=\partial_{x}^{2}+\partial_{y}^{2}$. This norm can easily be calculated by weighting the standard $L^{2}$-norm in Fourier space by an additional term $\left(1+\|\vec{k}\|^{2}\right)^{1 / 2}$. It penalizes high frequencies where the penality is a polynomial with degree $l$ in the distance to the k-space center. To achieve the desired regularization, the operator and the representation of the coil profiles are transformed with a preconditioning matrix $W$ which contains a Fourier transform of the coil part multiplied by a diagonal weighting matrix

$$
\begin{aligned}
& \left(\begin{array}{c}
\rho \\
\hat{c}_{1} \\
\vdots \\
\hat{c}_{N}
\end{array}\right) \\
& \quad=\left(\begin{array}{cccc}
\mathrm{I} & & & \\
& \left(1+\|\vec{k}\|^{2}\right)^{I / 2} \mathcal{F} & & \\
& & \ddots & \\
& & & \left(1+\|\vec{k}\|^{2}\right)^{I / 2} \mathcal{F}
\end{array}\right)\left(\begin{array}{c}
\rho \\
C_{1} \\
\vdots \\
c_{N}
\end{array}\right) .
\end{aligned}
$$

The IRGNM algorithm is then applied to the following transformed but equivalent system of equations

$$
\begin{aligned}
\hat{x} & =W^{-1} x \\
G \hat{x} & =F W \hat{x}=y .
\end{aligned}
$$

Using these definitions the new minimization problem-to be solved in each Newton step-is given by

$$
\left\|D G\left(\hat{x}_{n}\right) d \hat{x}-\left(y-G\left(\hat{x}_{n}\right)\right)\right\|^{2}+\alpha_{n}\left\|\hat{x}_{n}+d \hat{x}-\hat{x}_{0}\right\|^{2} .
$$

A closer look at the regularization term reveals the effect of the above modification (assuming $x_{0}=\overrightarrow{0}$ for simplicity)

$$
\begin{aligned}
\left\|\hat{x}-\hat{x}_{0}\right\|^{2} & =\left\|W^{-1} x-W^{-1} x_{0}\right\|^{2} \\
& =\|\rho\|^{2}+\sum_{j=1}^{N}\left\|\hat{c}_{j}\right\|^{2} \quad \text { using } \quad x_{0}=\overrightarrow{0} \\
& =\|\rho\|^{2}+\sum_{j=1}^{N}\left\|\left(1+\|\vec{k}\|^{2}\right)^{l / 2} \mathcal{F} c_{j}\right\|^{2} \\
& =\|\rho\|^{2}+\sum_{j=1}^{N}\left\|c_{j}\right\|_{l}^{2} .
\end{aligned}
$$

The $L_{2}$-norm used in the regularization of the coil part of the transformed system therefore corresponds to the Sobolev norm in the original space, penalizing high frequencies and enforcing the smoothness of the coil sensitivities. Flow charts for the calculation of the modified operator, the derivative, and its adjoint are shown in Fig. 1.

\section{Postprocessing}

As mentioned earlier, the system of equations is underdetermined even in the fully sampled case. Although this ambiguity may be removed by the choice of the regularization terms, the result is not necessarily identical to a typical sum-of-squares reconstruction. This difference manifests itself as slight changes in the large-scale intensity distribution. By multiplying the resulting image with the root of the sum of squares of the estimated coil sensitivities and 

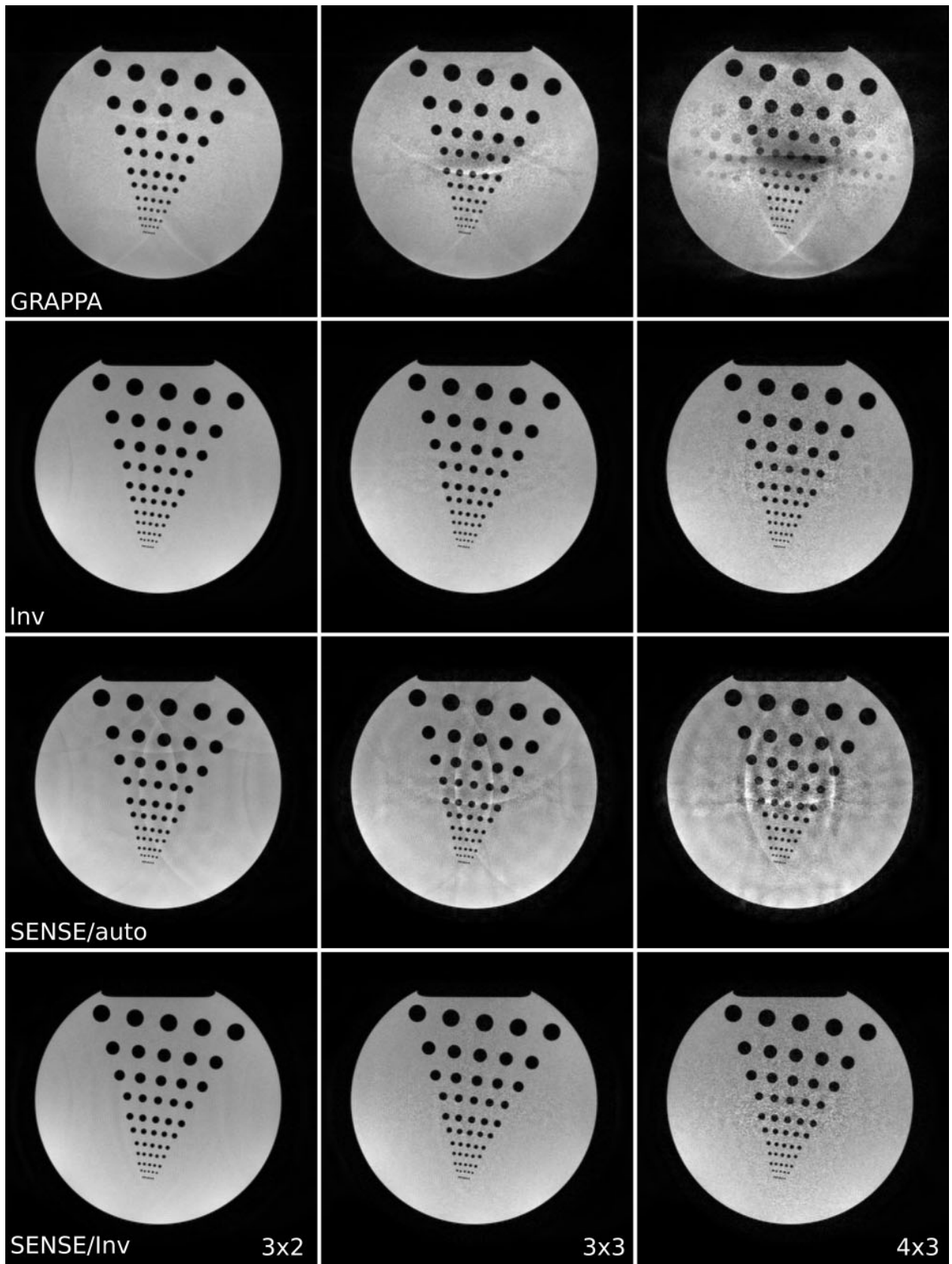

FIG. 2. 3D FLASH MRI partitions of a phantom reconstructed with GRAPPA, autocalibrated SENSE (SENSE/auto), nonlinear inversion (Inv) and SENSE with coil sensitivities taken from nonlinear inversion (SENSE/Inv) for 2D acceleration factors of $6=3 \times 2,9=3 \times 3$, and $12=4 \times 3$ using $24 \times 24$ reference lines and 16, 19, and 21 iterations of the nonlinear inversion algorithm, respectively.

dividing the coil sensitivities by the same quantity, this difference can be removed in a simple step

$$
\rho_{\text {final }}=\rho \cdot \sqrt{\sum_{j=1}^{N}\left|c_{j}\right|^{2}} .
$$

Although not strictly necessary, this postprocessing is useful when comparing images or coil sensitivities to images reconstructed with different regularization parameters, other parallel imaging algorithms, or from fully sampled data sets.

\section{MATERIALS AND METHODS}

Experimental examples were obtained for a water phantom and the brain of healthy volunteers at 2.9 T (Siemens Magnetom TIM Trio, Erlangen, Germany) using a 12-channel head coil. Written informed consent was obtained from all subjects prior to the examination. 

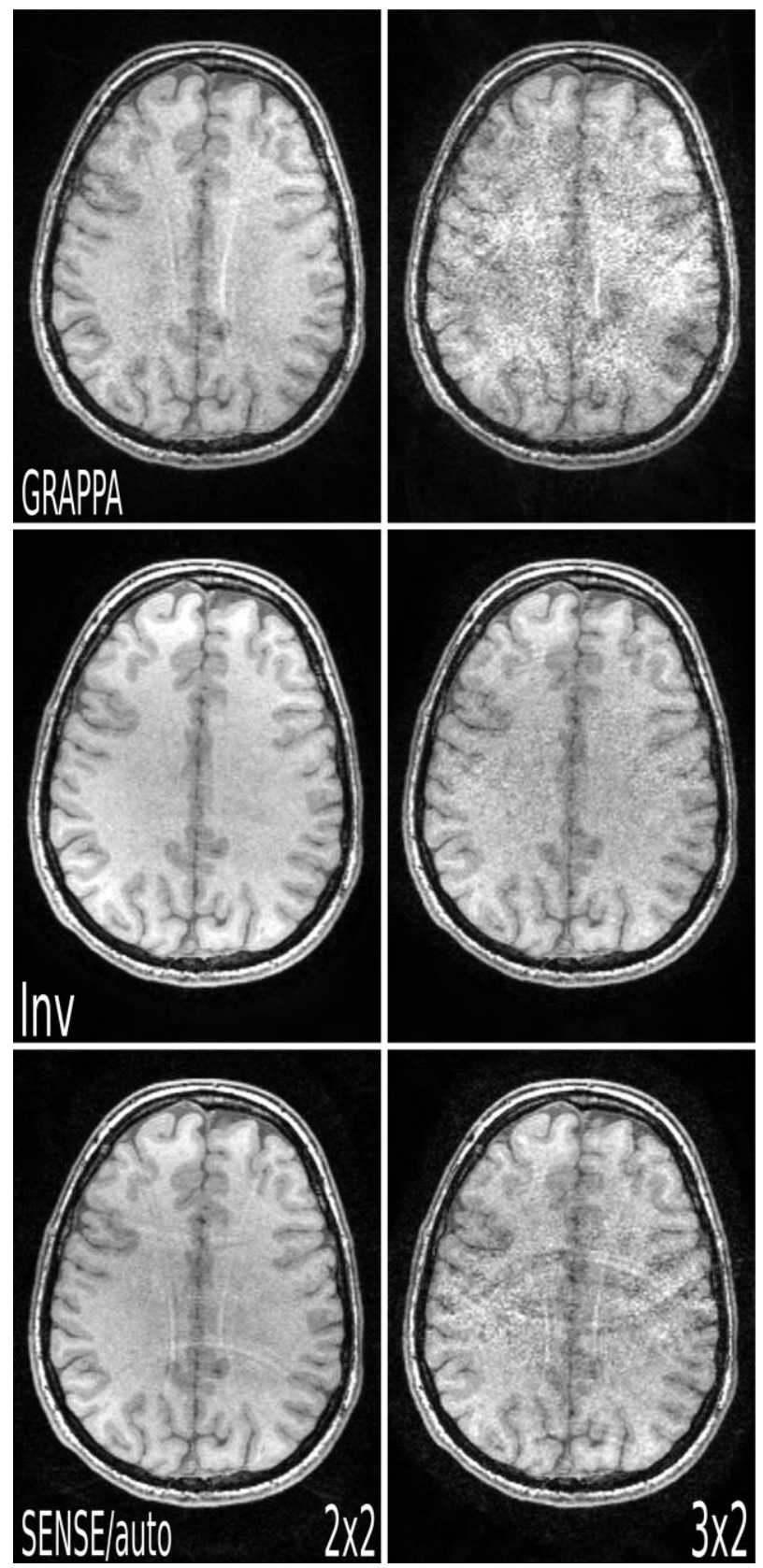

FIG. 3. 3D FLASH MRI partitions of the human brain reconstructed with GRAPPA, autocalibrated SENSE and nonlinear inversion (Inv) for $2 \mathrm{D}$ acceleration factors of $4=2 \times 2$ and $6=3 \times 2$ using $16 \times 16$ reference lines using 14 and 18 iterations, respectively.

The proposed algorithm was implemented in a C program using the FFTW3 library. Raw data was acquired, reconstructed offline, and the results were compared with the GRAPPA algorithm implemented on the MRI system (software version: VB13) and to an autocalibrated version of SENSE. As confirmed by personal communication the GRAPPA implementation is based on the algorithm presented in (10) but contains unpublished proprietary modifications. It may still serve as a valid reference because it is widely distributed and its characteristics are documented in numerous studies about parallel imaging methods and applications. The SENSE algorithm used here relies on the autocalibration technique from (9)-including an apodization with the Kaiser window $(\beta=4)$-and employs an iterative (CG) SENSE reconstruction (4) for image reconstruction. This generic SENSE version was chosen, because it allows for the inclusion of reference lines in the reconstruction - similar to GRAPPA and the proposed nonlinear inversion algorithm.

Images were acquired with use of a 3D RF-spoiled FLASH sequence $\left(\mathrm{TR} / \mathrm{TE}=10.6 / 4.2 \mathrm{~ms}\right.$, flip angle $\left.=17^{\circ}\right)$ with an isotropic spatial resolution of $1 \mathrm{~mm}$ spin-echo sequence with two groups of five echoes each. Parallel imaging was performed with variable acceleration (undersampling) factors in all phase-encoding directions and with variable numbers of reference lines. To improve the SNR of the 3D gradient-echo images of the human brain, the acquisitions employed two accumulations. For demonstration purposes individual partitions were selected from the 3D data orthogonal to the readout direction, that is after 1D Fourier transformation along the frequency-encoding axis. These 2D partitions were extracted and subsequently reconstructed using the proposed algorithm.

\section{RESULTS}

Figure 2 shows 3D MRI partitions of a water phantom obtained for $2 \mathrm{D}$ acceleration factors of $6=3 \times 2,9=3 \times 3$, and $12=4 \times 3$ using $24 \times 24$ reference lines. All acquisitions employed the 12 channels of the head coil and the images were reconstructed with GRAPPA, autocalibrated SENSE and nonlinear inversion, respectively. Visual inspection reveals that GRAPPA and autocalibrated SENSE lead to ghosting artifacts for a $2 \mathrm{D}$ acceleration factor of 6 that get increasingly worse for higher degrees of undersampling. In contrast, the proposed nonlinear inversion method is able to reconstruct almost artifact-free images up to the theoretical maximum of 12, although at this level the quality in central areas of the image begins to deteriorate. The SENSE reconstructions in the bottom part of Fig. 2 demonstrate that the high quality of the nonlinear reconstructions is primarily due to an improved estimation of the sensitivity profiles. SENSE reconstructions achieve a similar quality if the coil sensitivities are not estimated from the reference lines but taken from the calculations of the nonlinear inversion algorithm.

The above findings are confirmed for in vivo conditions. GRAPPA, autocalibrated SENSE and nonlinear inversion reconstructions for 3D MRI partitions of the human brain are compared in Fig. 3 for 2D acceleration factors of $4=$ $2 \times 2$ and $6=3 \times 2$ using $16 \times 16$ reference lines. Again, GRAPPA and autocalibrated SENSE images yield ghosting artifacts and considerably more noise than reconstructions by nonlinear inversion (using 14 and 18 iterations). For the nonlinear inversion reconstruction with 2D acceleration factor $2 \times 2$, Figs. 4-6 detail the extreme smoothness of the estimated coil sensitivities as well as the influence of the total number of iterations and the number of iterative updates of the coil information, respectively. The progressive reduction of the undersampling artifacts by increasing the number of iterations from 11 to 14 is demonstrated in Fig. 5. Complementary, Fig. 6 supports the notion that the improved reconstructions by nonlinear inversion are 

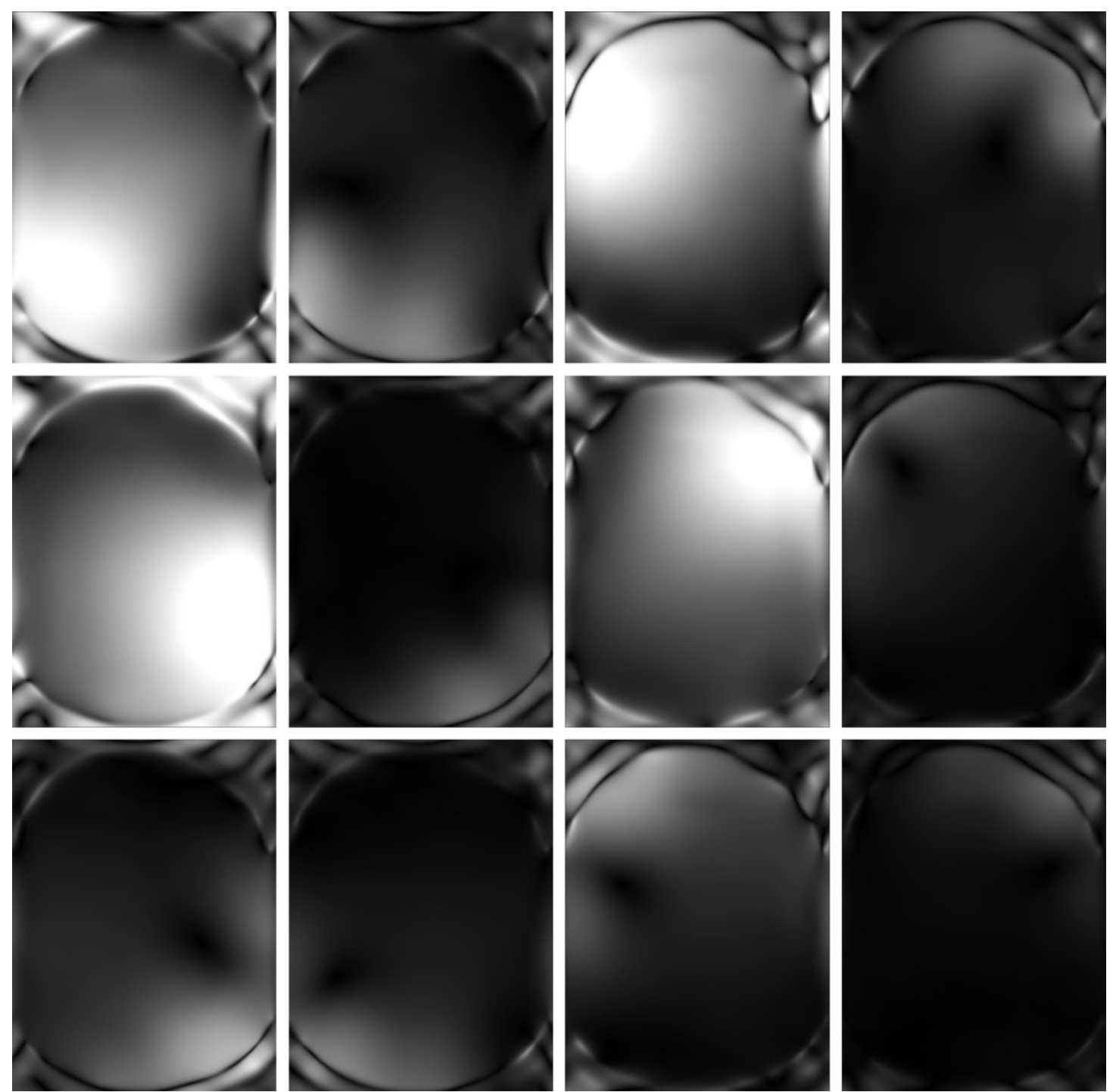

FIG. 4. Coil sensitivities corresponding to the Inv reconstruction (2D acceleration factor 4, 14 iterations) shown in Fig. 3.

largely due to the improved estimation of the coil sensitivities. When limiting the update of the coil information to $8,10,12$, or 14 iterations, the remaining iterations of the image information (up to 14) refer to a linear inversion similar to CG-SENSE. The comparison of respective reconstructions in Fig. 6 clearly indicates that an early termination of the coil update leaves the rest of the iterations with slightly incorrect coil sensitivities which in turn give rise to residual ghosting artifacts in the final image. Noteworthy, because these artifacts are related to errors in the coil sensitivities and not due to the regularization, they cannot be eliminated by adding further iterations without including an update of the coil information.

The relevance of the number of reference lines is examined in Fig. 7 which compares 3D FLASH MRI partitions of the human brain reconstructed with GRAPPA, autocalibrated SENSE and nonlinear inversion (2D acceleration factor 4) for $24 \times 24,16 \times 16$, and $8 \times 8$ reference lines. Although reconstructions by nonlinear inversion exhibit no ghosting artifacts and only a moderate increase of central noise for the lowest number of reference lines, GRAPPA and SENSE reconstructions are much more sensitive to accurate estimations of the coil information from a sufficiently high number of reference lines. Severe undersampling artifacts occur for both $16 \times 16$ and $8 \times 8$ reference lines.

\section{DISCUSSION}

This work introduces a new reconstruction method for autocalibrated parallel imaging which is based on regularized nonlinear inversion. The approach allows for a simultaneous calculation of the unknown coil sensitivity maps and the unknown spin density of the object using all available data. At least for the experimental conditions examined here, that is $2 \mathrm{D}$ acceleration factors of up to 12 , reference lines as few as $8 \times 8$, and conventional 2D and 3D MRI sequences, the proposed strategy yields images with visually reduced artifacts compared with the two-step approaches GRAPPA and autocalibrated SENSE. These methods first estimate information about the coil 

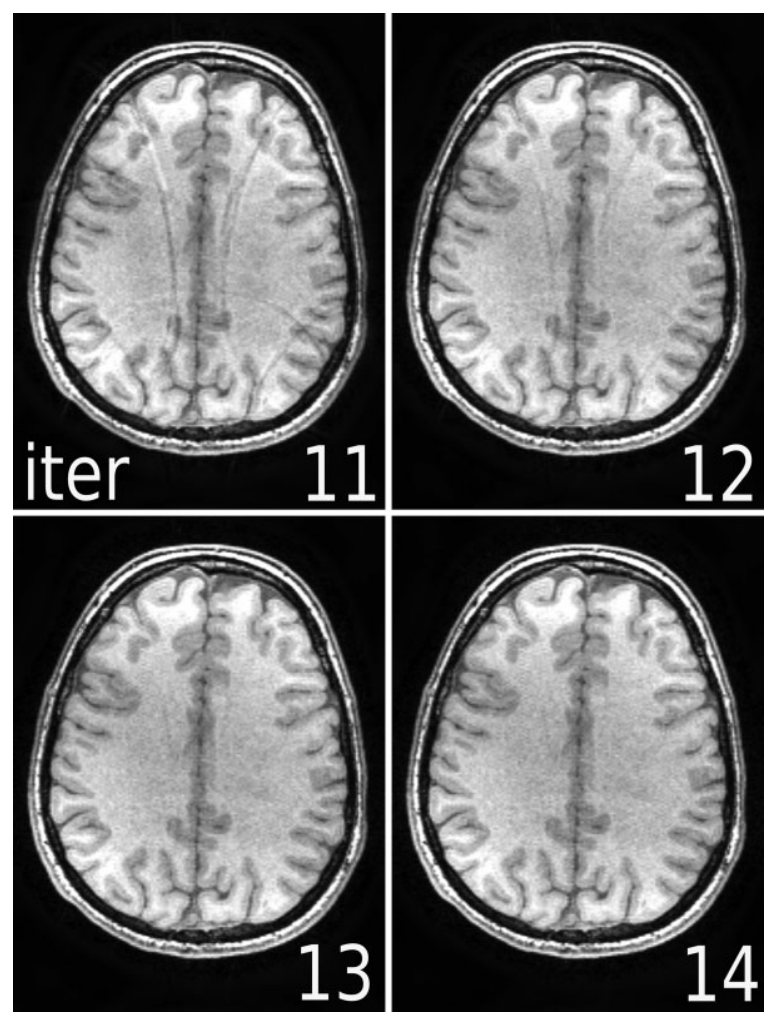

FIG. 5. Influence of the number of iterations (11-14) on the Inv reconstruction (2D acceleration factor 4 ) shown in Fig. 3.

sensitivities (which in the case of GRAPPA is encoded in the reconstruction weights) from only a part of the data and then solve a linear equation where the coil information remains fixed.

The necessity to improve the estimation of the coil sensitivity profiles in parallel image reconstructions has also been recognized by others. As mentioned before, it has recently been proposed to exploit the bilinear structure of the MRI signal equation to solve the system of equations for the coil profiles and object functions in an alternating way (1). In comparison to the alternating minimization scheme, an advantage of the Newton methods used here is a greater flexibility for incorporating additional nonlinear constraints and regularization terms. Such options will be even more important for higher acceleration factors that suffer from increased noise amplification. A Newton-type method has also been used for parallel MRI in (13), but the algorithm presented there computes the full Jacobian $D F\left(x_{n}\right)$ and uses a QR decomposition to solve the regularized Newton equations. Therefore, the time and memory complexity is much higher compared to the method presented here. Moreover, the use of a small number of basis functions, which ensures the smoothness of the coil profiles in (1) and (13) seems problematic because it limits the accuracy of the profile reconstructions as opposed to the use of Sobolev norms.

\section{Choice of Parameters}

The parameters for the proposed nonlinear inversion algorithm were chosen as follows: As initial guess $x_{0}$ the object function $\rho$ was set to constant 1 and the coil profiles $c_{1}, \ldots, c_{N}$ to zero. The Sobolev index for the regularization of the coil profiles was $l=16$. The first regularization parameter was chosen as $\alpha_{0}=1$ and reduced by a factor $q=2 / 3$ in each Newton step. To our experience the final results are not sensitive to the choice of any of these parameters. The only critical value is the regularization parameter $\alpha_{n_{*}}$ at the stopping index $n_{*}$, and hence the stopping criterion for the Newton method. The choice of $n_{*}$ is a trade-off between small noise (small $n_{*}$ ) and small undersampling artifact (large $n_{*}$ ).

Although the choice of the stopping index has been studied intensively in the literature on inverse problems, and a number of methods such as Morozov's discrepancy principle (12) or Lepskii's balancing principle (14) are available, no satisfactory solution for the problem has been described to the best of our knowledge. Part of the reason is that for images the $L^{2}$ error does not accurately describe the visual quality of a reconstruction. For the data presented here, the stopping index was therefore chosen by visual inspection. Although it may be fixed for repeated measurements with the same MRI protocol, the automatic determination of a robust stopping criterion is certainly a most desirable feature but outside the scope of this work.

It should be noted that a choice of the right regularization parameters is not unique to the present algorithm but a general problem common to all parallel imaging methods. Even some of the GRAPPA or SENSE images shown in this work could probably be improved by manually optimizing the regularization parameters (chosen by the vendor). Nevertheless, a simple adjustment of the GRAPPA and SENSE regularization would not be enough to generate images
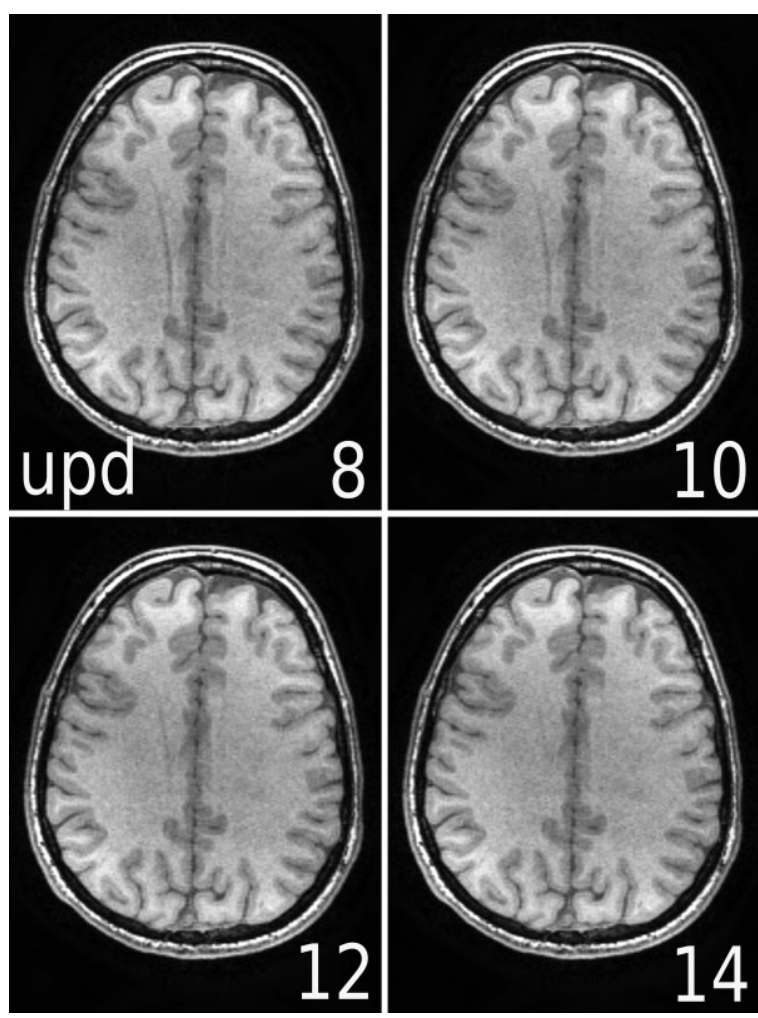

FIG. 6. Influence of the number of coil updates (8 to 14) on the Inv reconstruction (2D acceleration factor 4, 14 iterations) shown in Fig. 3. 

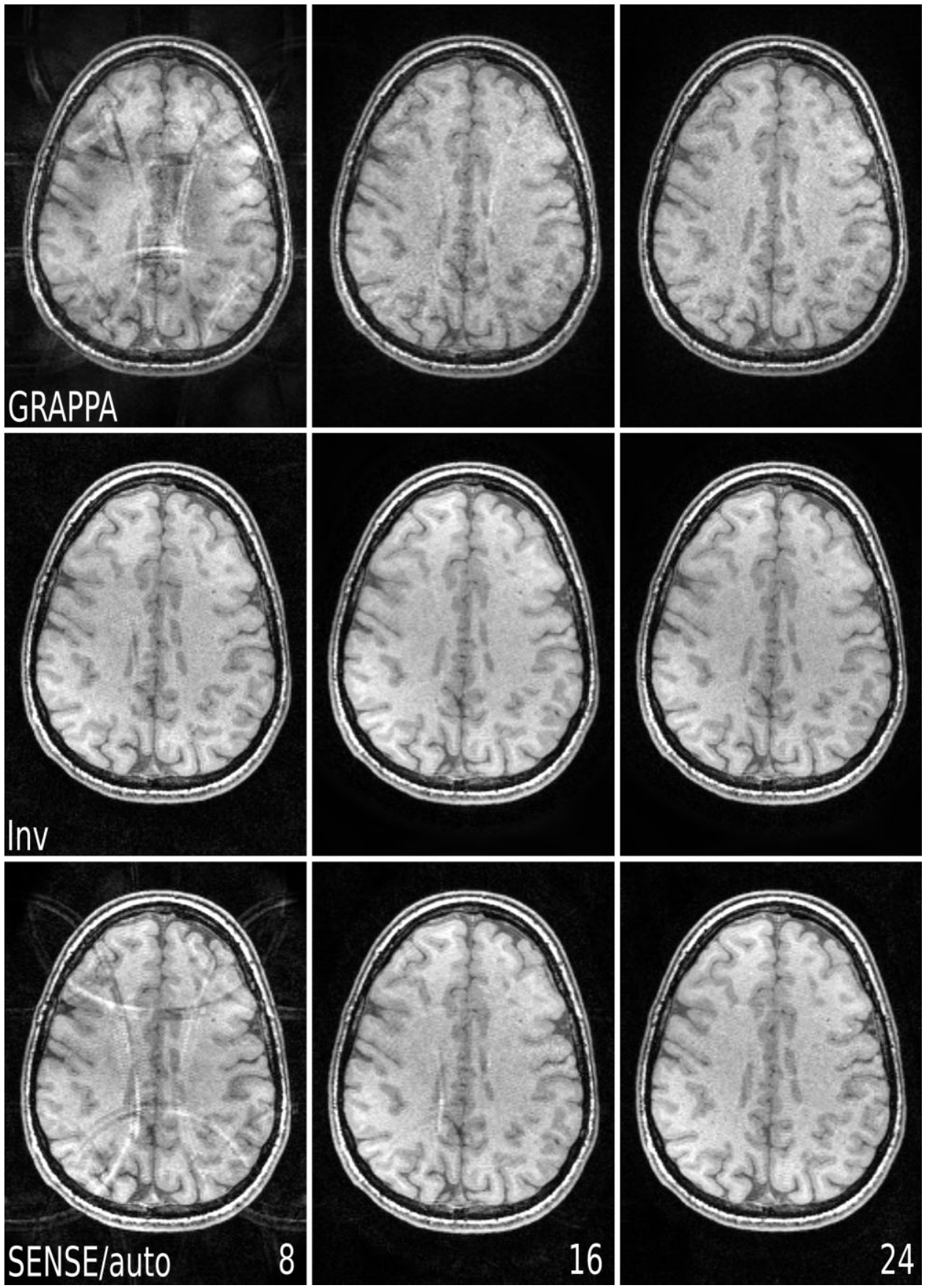

FIG. 7. 3D FLASH MRI partitions of the human brain reconstructed with GRAPPA, autocalibrated SENSE and nonlinear inversion (Inv) for a $2 \mathrm{D}$ acceleration factor of 4 using $24 \times 24,16 \times 16$, and $8 \times 8$ reference lines with 14 and $22(8 \times 8)$ iterations.

equivalent to those obtained by nonlinear inversion. For example, pronounced qualitative differences are demonstrated for reconstructions with a low number of reference lines. As demonstrated in Fig. 7 respective GRAPPA reconstructions exhibited both high noise and residual ghosting artifacts, whereas nonlinear inversion resulted in very much improved images. Because the overall regularization determines the tradeoff between noise and artifacts, the advantage of the proposed algorithm relative to GRAPPA cannot be explained by a better choice of regularization parameters.

\section{Convergence}

For Newton-type algorithms convergence is not automatically guaranteed and in some cases even requires an initial guess close to the solution. In practice, this seems to be no problem for the proposed algorithm. For example, in situations where GRAPPA produces a reasonable image, the nonlinear inversion algorithm decreases the residual in each iteration yielding a good solution after only $10-25$ iterations - even for very low numbers of reference lines. In all these cases, the initial guess was always choosen 
as described above. In extreme situations, that is without any reference lines or for acceleration factors greater than the number of receiver channels, the algorithm is still able to decrease the residual but without generating reasonable images. Very slow convergence in the sense that the norm of the residual decreases very slowly is only observed when the image object is completely empty and the algorithm tries to fit only noise. This case could be dealt with by calculating the amount of energy in the data before starting the iteration.

The theoretical treatment of convergence is complicated by the fact, that parallel imaging is increasingly worse conditioned for higher acceleration factors. This holds true even in the linear case and for perfectly known coil sensitivities. The exact solution of the system is very far from the desired solution, because of the amplified noise. Every parallel imaging algorithm should therefore contain some form of regularization. For the proposed algorithm this is accomplished by terminating the iteration long before convergence to a undesirable solution is reached. In fact, it would be advantageous to replace the assessment of convergence by a different mathematical criterion. It might quantify the ability of the algorithm in conjunction with its automatic stopping criterion (e.g., the discrepancy principle) to produce a series of solutions for input vectors with decreasing noise that converges to the exact solution of the noiseless case. However, a formal proof of this property was not yet attempted for the proposed algorithm.

\section{Computational Speed}

In the present implementation, the reconstruction of a single $256 \times 256$ image by nonlinear inversion takes less than a minute on a modern processor. Although this is too slow for applications to a complete 3D MRI data set, significant acceleration can already be expected by simple measures such as a multithreaded implementation utilizing multiple processor cores and switching from double to single precision floating point numbers. Further possibilities include preconditioning techniques (e.g., the method presented in (15)), which would reduce the number of conjugate gradient iterations, and adaptive discretization in particular of the coil profiles, which would reduce the cost of the first Newton steps by an order of magnitude.

\section{CONCLUDING REMARKS}

The formulation of the MRI reconstruction for multiple receiver coils as a nonlinear inversion problem and its application to autocalibrated parallel imaging has been demonstrated to markedly improve the achievable image quality for high acceleration factors when compared to conventional methods.

Although the extension of the reconstruction process to a nonlinear system of equations seems to represent a large complication, it opens a number of advantageous possibilities because of a simple and unified way to incorporate a priori knowledge by choosing appropriate norms. For example, by including a gridding operator the approach should be directly applicable to self-calibrating noncartesian sampling schemes. Another possibility would be to more accurately model the acquisition process by jointly estimating relaxation or field maps together with the coil sensitivities and the image content. A further extension already under investigation is the integration of total variation-based regularization which combats the high noise for higher acceleration factors and-in combination with appropriate sampling patterns-would allow for the benefits of reduction factors higher than the number of receiver coils as explained by the compressed sensing theory $(16,17)$. In fact, the move to more general nonlinear methods seems to be the next logical step in MRI reconstruction.

\section{REFERENCES}

1. Ying L, Sheng J. Joint image reconstruction and sensitivity estimation in SENSE (JSENSE). Magn Reson Med 2007;57:1196-1202.

2. Hoge WS, Brooks DH, Madore B, Kyriakos WE. A tour of accelerated parallel MR imaging from a linear systems perspective. Concepts Magn Reson Part A 2005;27:17-37.

3. Pruessmann KP, Weiger M, Scheidegger MB, Boesiger P. SENSE: Sensitivity encoding for fast MRI. Magn Reson Med 1999;42:952-962.

4. Pruessmann KP, Weiger M, Bornert P, Boesiger P. Advances in sensitivity encoding with arbitrary k-space trajectories. Magn Reson Med 2001;46:638-651.

5. Kyriakos WE, Panych LP, Kacher DF, Westin CF, Bao SM, Mulkern RV, Jolesz FA. Sensitivity profiles from an array of coils for encoding and reconstruction in parallel (SPACE RIP). Magn Reson Med 2000;44:301308.

6. Sodickson DK, Manning WJ. Simultaneous acquisition of spatial harmonics (SMASH): Fast imaging with radiofrequency coil arrays. Magn Reson Med 1997;38:591-603.

7. Bydder M, Larkman DJ, Hajnal JV. Generalized SMASH imaging. Magn Reson Med 2002;47:160-170.

8. Griswold MA, Breuer F, Blaimer M, Kannengiesser S, Heidemann RM, Mueller M, Nittka M, Jellus V, Kiefer B, Jakob PM. Autocalibrated coil sensitivity estimation for parallel imaging. NMR Biomed 2006;19:316324.

9. McKenzie CA, Yeh EN, Ohliger MA, Price MD, Sodickson DK. Selfcalibrating parallel imaging with automatic coil sensitivity extraction. Magn Reson Med 2002;47:529-538.

10. Griswold MA, Jakob PM, Heidemann RM, Nittka M, Jellus V, Wang J, Kiefer B, Haase A. Generalized autocalibrating partially parallel acquisitions (GRAPPA). Magn Reson Med 2002;47:1202-1210.

11. Bakushinsky AB, Kokurin MY. Iterative methods for approximate solution of inverse problems. Dordrecht: Springer; 2004.

12. Engl HW, Hanke M, Neubauer A. Regularization of inverse problems. Dordrecht, Boston, London: Kluwer Academic Publisher; 1996.

13. Bauer F, Kannengiesser S. An alternative approach to the image reconstruction for parallel data acquisition in MRI. Math Methods Appl Sci 2007;30:1437-1451.

14. Bauer F, Hohage T. A Lepskij-type stopping rule for regularized Newton methods. Inverse Problems 2005;21:1975-1991.

15. Hohage T. On the numerical solution of a three-dimensional inverse medium scattering problem. Inverse Problems 2001;17:1743-1763.

16. Donoho D. Compressed sensing. IEEE T Inform Theory 2006;52:12891306.

17. Candes E, Romberg J, Tao T. Robust uncertainty principles: Exact signal reconstruction from highly incomplete frequency information. IEEE T Inform Theory 2006;52:489-509. 